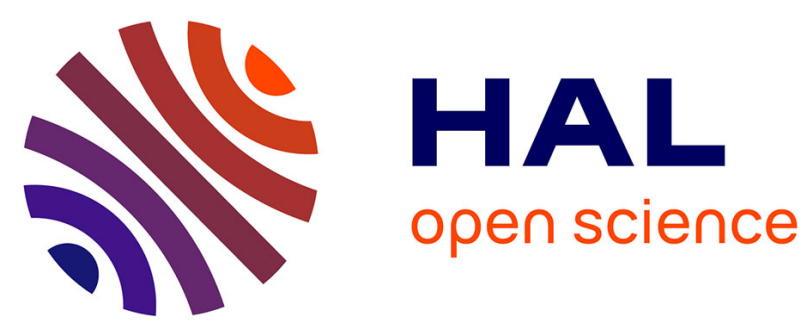

\title{
Coronary Vein Extraction in MSCT Volumes Using Minimum Cost Path and Geometrical Moments
}

Marie-Paule Garcia, Jérôme Velut, Dominique Boulmier, Christophe Leclerc, Mireille Garreau, Pascal Haigron, Christine Toumoulin

\section{- To cite this version:}

Marie-Paule Garcia, Jérôme Velut, Dominique Boulmier, Christophe Leclerc, Mireille Garreau, et al.. Coronary Vein Extraction in MSCT Volumes Using Minimum Cost Path and Geometrical Moments. IEEE Journal of Biomedical and Health Informatics, 2013, pp.336 - 345. 10.1109/JBHI.2013.2245420 . inserm-00874947

\section{HAL Id: inserm-00874947 https://www.hal.inserm.fr/inserm-00874947}

Submitted on 21 Nov 2013

HAL is a multi-disciplinary open access archive for the deposit and dissemination of scientific research documents, whether they are published or not. The documents may come from teaching and research institutions in France or abroad, or from public or private research centers.
L'archive ouverte pluridisciplinaire HAL, est destinée au dépôt et à la diffusion de documents scientifiques de niveau recherche, publiés ou non, émanant des établissements d'enseignement et de recherche français ou étrangers, des laboratoires publics ou privés. 


\title{
Coronary Vein Extraction in MSCT Volumes using Minimum Cost Path and Geometrical Moments
}

\author{
M.-P. Garcia, J. Velut, D. Boulmier, C. Leclercq, M. Garreau, P. Haigron, C. Toumoulin
}

\begin{abstract}
This work deals with the extraction of patientspecific coronary venous anatomy in pre-operative Multi-Slice Computed Tomography (MSCT) volumes. A hybrid approach has been specifically designed for low-contrast vascular structure detection. It makes use of a minimum cost path technique with a Fast-Marching front propagation to extract the vessel centerline. A second procedure was applied to refine the position of the path and estimate the local radius along the vessel. This was achieved with an iterative multiscale algorithm based on geometrical moments. Parameter tuning was performed using a dedicated numerical phantom, then the algorithm was applied to extract the coronary venous system. Results are provided on three MSCT volume sequences acquired for patients selected for a Cardiac Resynchronisation Therapy (CRT) procedure. A visibility study was carried out by a medical expert who labelled venous segments on a set of eighteen volumes. A comparison with two other FastMarching techniques and a geometrical moment based tracking method is also reported.
\end{abstract}

Index Terms-Coronary veins, MSCT, 3D vascular extraction, Minimum cost path, Fast Marching, Geometrical moments

\section{INTRODUCTION}

Cardiac resynchronization therapy (CRT) is assessed as an efficient therapeutic approach for patients who suffer from severe heart failure, sinus rhythm and ventricular conduction delay [1]. The procedure consists in pacing simultaneously or with a small delay, both the right ventricle (RV) and the left ventricle (LV). Daubert et al. proposed a totally transvenous approach [2] using three pacing leads. Two are respectively positioned in the right atrium $\left(l_{3}\right)$ and the $\mathrm{RV}\left(l_{2}\right)$ through the vena cava while the third one $\left(l_{1}\right)$ is inserted through the coronary sinus into a venous branch that runs along the free wall of the left ventricle (see Fig. 1). The major issue is the placement of the LV lead. Indeed, the implantation of the pacing devices is currently $2 \mathrm{D}$ venous $\mathrm{X}$-Ray coronarography guided and the implant success rate is limited to $85-92 \%$ [3]. The main reasons of failure are the difficulty of accessing the candidate veins, incorrect or suboptimal pacing site selection and possible electrode displacements, as well as the risk of dissecting the coronary sinus. Thus, an adequate planning is needed to improve and secure the implant procedure. Our work

This work is supported by the French Research Minister (n 04 T 187 $188-189-190 \mathrm{CITH}$ and $\mathrm{n}^{\circ}$ ANR-07-EMPB-007-01), the Brittany region and the European Community's Seventh Framework Programme (FP7/20082012) under grant agreement $n^{\circ} 224495$ (euHeart project).

M.-P. Garcia, C. Toumoulin, P. Haigron, J. Velut, M. Garreau are with INSERM, UMR 1099, Rennes, F-35000, France and Université de Rennes 1, LTSI, Rennes, F-35000, France;

C. Leclercq, D. Boulmier are with INSERM, UMR 1099, Rennes, F-35000, France and Université de Rennes 1, LTSI, Rennes, F-35000, France and CHU Rennes, Service de cardiologie et maladies vasculaires, Rennes, F-35000, France;

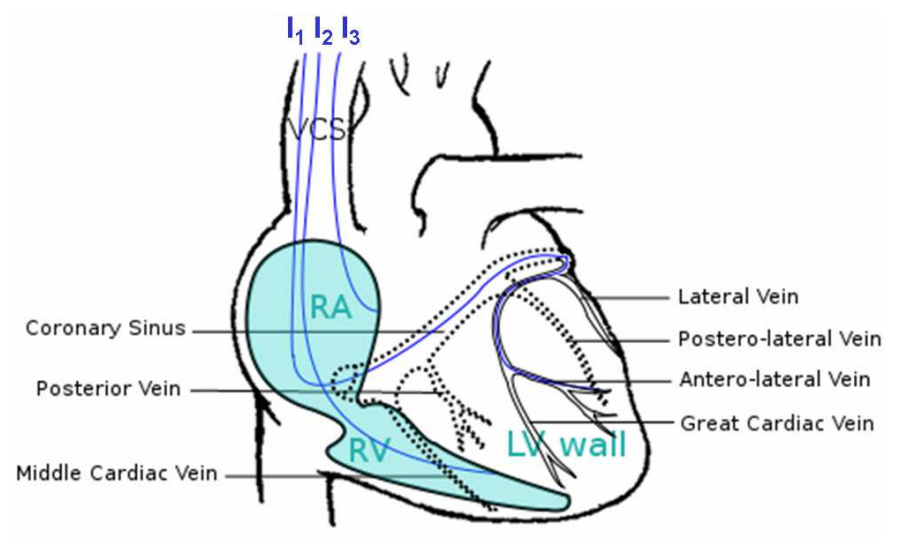

Fig. 1. Lead implantation for CRT and coronary venous anatomy on frontal view of the heart.

deals with the description of patient-specific coronary venous anatomy to define pre-operatively the target vein, plan the best path to reach it and confirm its accessibility.

The study is carried out using contrast-enhanced MutliSlice Computed Tomography (MSCT) data. This modality is considered as a reliable technique to depict the cardiac venous system [4]. Nevertheless, MSCT scans are tailored for optimal visualization of the coronary arteries. Indeed, the dye product passes first through the coronary arteries before being gathered by the coronary venous tree, which lead to suboptimal enhancement of the coronary veins, especially of second and third degree side branches having a small diameter. More generally, the dye product appears diluted when reaching the venous network, resulting in a weak contrast and variable appearance along the vessels with hypodense zones and blurred contours. Another difficulty relates to the close proximity of structures having similar contrast, i.e., cavities and coronary arteries. In addition, the venous anatomy tends to be highly variable from one patient to another [5], i.e., number of branches, location and length. Thus, a primary goal towards planning is to provide an efficient method for extracting coronary veins in MSCT volumes.

A lot of work has been devoted to the extraction of vascular networks, but a large majority concerns coronary arteries in Magnetic Resonance Imaging (MRI) and MSCT (see [6], [7], [8] for the main surveys). The methods can be categorized in different ways (the application [6], the mathematical framework [7] or the extraction scheme [8]) according to the issue to be highlighted. If we consider the extraction scheme, three main families are reported in the literature: regiongrowing-based approaches [9], active contour family [10], [11] 
and centerline extraction methods. The latter gathers direct centerline tracking [12], [13], model-based [14] and minimum cost path techniques [15], [16], [17], [18]. The performances of these methods vary according to multiple factors: the presence of severe pathologies (acute stenosis, large aneurysm), local complexity of vascular networks (complicated branching situations) and the surrounding tissue environment. A first standardized evaluation methodology and reference database has been set up, within the 2008 MICCAI challenge, to quantitatively evaluate the coronary artery centerline extraction algorithms [19]. This evaluation does not include coronary venous structures.

The method, which is described here, aims to take into account the presence of nearby contrasted artery vessels and strong contrast changes along the veins. It makes use of a two-stage process. A minimal path technique is first applied to extract vein centerlines [20]. A new cost function has been designed that combines the multiscale Frangi's filter response with the direction of the front with respect to the vessel orientation in order to constrain its propagation direction and better handle contrast inhomogeneity and weakness inside the veins as the presence of nearby blood vessels. The extracted path does not always correspond to the vessel central axis. We applied then an iterative multiscale recentering process to shift it towards the central axis position and estimate the local radius along the vascular branch. This stage was carried out using geometrical moments under the hypothesis that the vessel can be locally modeled by a cylinder. Section II describes this algorithm. Parameter tuning was carried out on a numerical phantom. The set of experiments are described in Section III. Section IV finally provides some preliminary results on the coronary vein extraction with a qualitative evaluation and a comparison with three other methods dedicated to centerline extraction.

\section{MethoD}

This section introduces our vessel extraction algorithm designed for coronary veins into MSCT data sets.

\section{A. Minimum Cost Path Computation}

Minimum cost path techniques aim to find a curve from a source point $P_{0}$ to a final point $P_{F}$ that minimizes an energy functional that is of the form:

$$
E(\gamma)=\int_{\gamma} \Lambda(\gamma(s)) d s
$$

where $\Lambda$ is an image-based measure and $\gamma$ is the path joining the two points. The value $\Lambda$ is given by a cost function that is defined to provide low values on the desired features (i.e. contour or centerline), the objective being to find a path along which the integral of $\Lambda$ is minimal. The solution of this minimization problem goes through the computation of an energy map $U(P)$ such as:

$$
U(P)=\inf _{C_{P_{0}, P}} E(\gamma)=\inf _{\gamma \in C_{P_{0}, P}}\left\{\int_{\gamma} \Lambda(\gamma(s)) d s\right\}
$$

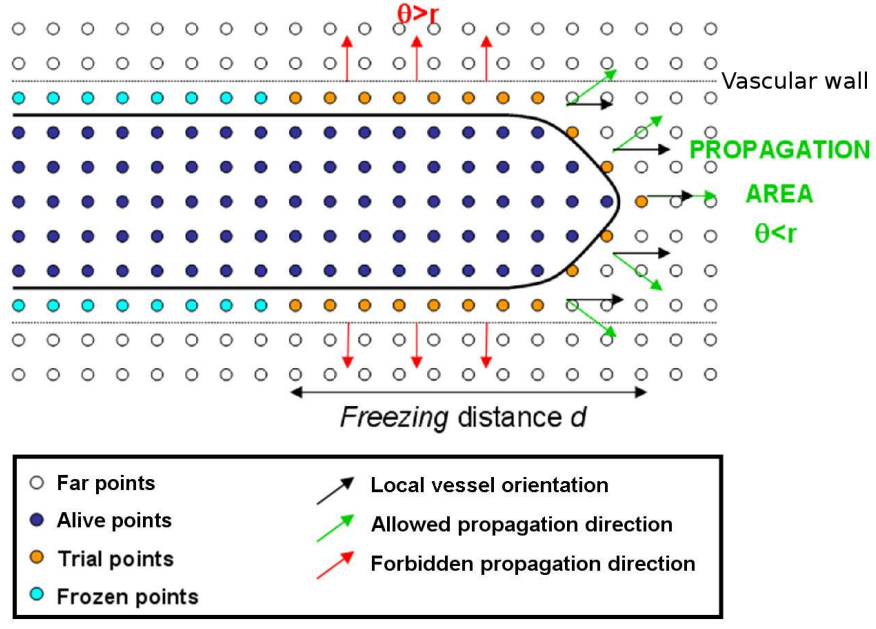

Fig. 2. The restricted and oriented front propagation and its combination with the Freezing procedure in 2D space. The front is only allowed to propagate into a restricted area defined by the angular value $r . \theta$ is the angle between the direction of the normal to the front $\vec{n}$ at position $(x, y, z)$ (red or green arrow) and the local orientation $\vec{\Gamma}$ (black arrow).

where $C_{P_{0}, P}$ is the set of all possible paths going from a source point $P_{0}$ to a given point $P$. To ensure that the map $U(P)$ has got only one minimum, we assumed that $\Lambda>0$. Then the minimum cost path can be retrieved by applying a gradient descent algorithm on the map $U$ from $P_{F}$ to $P_{0}$.

1) Fast Marching Algorithm: The computation of the energy map $U(P)$ is solved considering a Hamiltonian approach. We aim at computing the travel time $U$ that satisfies the Eikonal equation:

$$
\|\nabla U\|=\Lambda
$$

The Fast Marching algorithm [11] is an efficient way to solve the previous equation. It consists in a front which starts its propagation from the point $P_{0}$ and progresses over the image towards areas presenting low costs. Each time the front passes over the point $P_{x, y, z}$, its travel time $U_{x, y, z}$ is computed corresponding to the cumulative travel cost of the minimum cost path from $P_{0}$ to $P_{x, y, z}$. In our approach, to deal with low-contrast environment, we constrained the front progression towards a privileged direction. We want the front not to propagate beyond vessel boundaries. Therefore, the computation of the energy map $U$ was restricted to a region assimilated to a narrow band around the front to fit the following constraints (see Fig. 2): (1) the direction of propagation was restricted into an area in the estimated local direction, (2) and into vessel regions, given by Frangi's filter response [21], (3) a freezing distance was applied to freeze the points located at the tail of the propagating front and avoid the front flowing over the boundaries [22], [17]. In this implementation, the bifurcations of the coronary venous tree are not handled.

The algorithm is introduced in Table I and is referred as method $(\mathrm{VM}+\mathrm{OC})$ later on.

2) Cost function definition: The front propagation is guided by the following cost function which considers the reciprocal of the normalized vesselness measure $F_{x, y, z}$ weighted by 
orientation information $\rho$ :

$$
\begin{gathered}
\Lambda_{x, y, z}= \begin{cases}\left(\frac{1}{F_{x, y, z}} \cdot \rho\right)^{2} & \text { if } \theta<r \text { and } F_{x, y, z}>s \\
C, \quad & \text { otherwise }\end{cases} \\
\text { with } \rho=\sin (\theta)
\end{gathered}
$$

$F_{x, y, z}$ corresponds to the optimal response given by the vesselness function $F(x, y, z, \sigma)$ at the scale $\sigma$ that copes with the vessel radius:

$$
F_{x, y, z}=\max _{\sigma_{\min } \leq \sigma \leq \sigma_{\max }} F(x, y, z, \sigma)
$$

with $F_{x, y, z, \sigma}$ at a scale $\sigma$ given by:

$F_{x, y, z}=\left\{\begin{array}{l}0, \text { if } \lambda_{2}>0 \text { or } \lambda_{3}>0 \\ \left(1-\exp \left(\frac{-R_{A}^{2}}{2 \alpha^{2}}\right)\right) \exp \left(\frac{-R_{B}^{2}}{2 \beta^{2}}\right)\left(1-\exp \left(\frac{-S^{2}}{2 \gamma^{2}}\right)\right)\end{array}\right.$

and:

with $R_{A}=\left|\frac{\lambda_{2}}{\lambda_{3}}\right|, R_{B}=\frac{\left|\lambda_{1}\right|}{\sqrt{\left|\lambda_{2} \lambda_{3}\right|}}$ and $S=\sqrt{\lambda_{1}^{2}+\lambda_{2}^{2}+\lambda_{3}^{2}}$

$\lambda_{1}, \lambda_{2}, \lambda_{3}$ are the eigenvalues of the Hessian, ordered according to their magnitude as $\left|\lambda_{1}\right| \leq\left|\lambda_{2}\right| \leq\left|\lambda_{3}\right|$. The vessel local orientation is given by the eigenvector corresponding to the smallest eigenvalue $\lambda_{1}$ at scale $\sigma$. The parameters $\alpha, \beta$ and $\gamma$ are used to control the sensitivity of the filter to deviations from the cylindrical shape. The $\alpha$ parameter controls the ratio $R_{A}$ which discriminates plate-like structure. The $\beta$ parameter controls the ratio $R_{B}$ which discriminates blob-like structure. The $\gamma$ parameter controls the measure $S$ which influences noise suppression.

In (4), $\theta$ is the angle between the direction of the normal to the front $\vec{n}$ and the mean local vessel orientation $\vec{\Gamma}$. $\vec{n}$ is computed from the discrete approximation proposed by Parker et al. in [23] and $\vec{\Gamma}$ is estimated from the previous $n$ alive points whose vesselness measure is high (threshold $\mu$ ), to avoid noise disturbance. $C$ is a penalty cost given to voxels located outside the propagation area and at non-vessel positions. This cost is set to a high value to stop the front evolution at considered locations $(C=10000)$. The variable $s$ is a threshold on the vesselness criterion, which is set to a very small value $(s=0.01)$ to remove the background. Finally, the variable $r$ is an angular threshold that is used to orientate the propagation of the front and prevent it from leaking through the vessel boundaries. Its value will be studied later on in Section III.

3) Freezing distance: The cost function is efficient to propagate the front around the vessel axis for points located at the head of the front. However, in case of low contrast with surrounding structures, the cost given to points close to contours may not be high enough to definitively stop the front propagation at those positions and the front may leak beyond boundaries while advancing in the vessel. Thus, in order to stop the eventual propagation of the front in segments already considered, a Freezing process is applied, as in Deschamps and Cohen [22], [17]. As schematized in Fig. 2, the points having the label 'Trial' which are located far away from the head of the front are labeled 'Frozen' according to a Freezing distance $d$.
ALGORITHM Minimum Cost Path Computation

Notations:

- Alive Set: Nodes reached by the front whose $U$-value will not be changed;

- Trial Set: Nodes having at least one Alive neighbour and their $U$-value already estimated;

- Far Set: Nodes whose $U$-value has never been computed;

- $F_{x, y, z}$ is the vesselness measure of the point $P_{x, y, z}$.

Interactive Initialization: The user points a seed point $P_{0}$ and a final point $P_{F}$ in the branch of interest and gives the initial propagation direction $\vec{v}$.

Fast Marching Front Propagation:

1) Initialization:

- Tag the point $P_{0}$ as Alive and set $U\left(P_{0}\right):=0$;

- Tag Far, all other points $P$ and set $U(P):=\infty$;

- Tag Trial, each neighbour $P_{x, y, z}$ of the point $P_{0}$ for which the direction of the normal to the front is close to the initial propagation direction $\vec{v}$ and set $U\left(P_{x, y, z}\right):=1 / F_{x, y, z}$

2) while $P_{F}$ is not reached and the Trial set is not empty

- Find $P_{\min }$, the Trial point with the lowest $U$-value;

- Tag $P_{\min }$ as Alive

- For each neighbour point $P_{x, y, z}$ of $P_{m i n}$, tagged as Far:

- Compute the cost $\Lambda_{P_{x, y, z}}$ (4);

- Compute $U\left(P_{x, y, z}\right)$ by solving the Eikonal equation (3)

- if $P_{x, y, z}$ is a Far point: Add it to the Trial set;

- if $P_{x, y, z}$ is a Trial point: Update $U\left(P_{x, y, z}\right)$ if the new value is smaller

- Freeze Trial points whose distance to the front 'head' is greater than the freezing distance $d$ (Freezing process).

Path Extraction: Compute a path from $P_{0}$ to $P_{F}$ by gradient descent on the travel time map $U$.

TABLE I

Minimum Cost PATH COMPUTATION USING A FAST-MARCHING Front PROPAGATION.

4) Path extraction: Once the final point $P_{F}$ is reached by the front, the path from $P_{0}$ to $P_{F}$ is computed using the travel time map $U$. A gradient descent is applied on the map $U$ from the point $P_{F}$. Each new point $P_{i}$ of the path is the one amongst the neighbours of the point $P_{i-1}$ presenting the smallest difference between $U_{i}$ and $U_{i-1}$.

\section{B. Path Centering and Radius Estimation}

This second procedure aims at refining the path extracted at the previous stage (see II-A) and estimating the local radius at each centred position (see Fig. 3 for an overview of the approach). Local vessel features can be estimated analytically from geometrical moments computed in a spherical window centered on the vessel central axis and that fit the vessel radius. To achieve this goal, we use an iterative multiscale algorithm [24] which alternatively moves the sphere towards its center of gravity, estimates the vessel radius $r$ and adjust the size of the sphere $R$ until convergence of the sphere radius to the vessel size. The center of gravity and the local vessel radius was estimated using the first order moments (analytical expressions can be found in [24]).

This algorithm is described in Table II. The extracted path from $P_{0}$ to $P_{F}$ consists of successive neighbouring points of the image. The path is subsampled to avoid the spherical window (in the iterative multiscale algorithm) converging towards identical positions for several neighbouring points. 


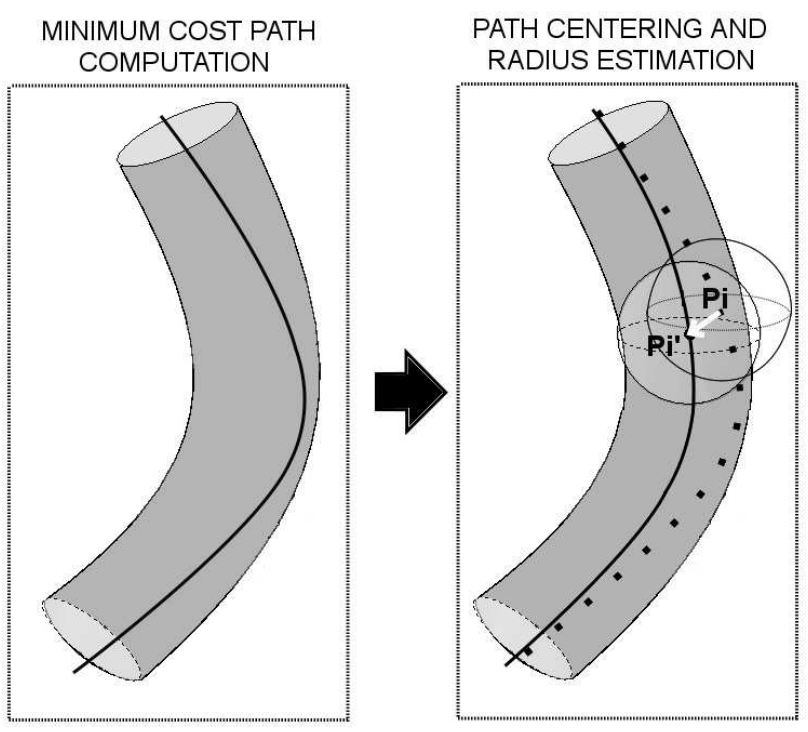

Fig. 3. Overview scheme of the proposed method: (Left) The minimum cost path computation yields a first estimation of the vessel centerline (algorithm Table I). (Right) The second procedure refines the centerline position and yields a radius estimation at each corrected position $P_{i}^{\prime}$. The previous path is subsampled and a spherical window centered on each position $P_{i}$ is moved towards the central axis and its size fits the local vessel radius (algorithm Table II).

For each point of the subsampled path $P_{i}$, given an initial spherical window size $R_{0}^{i}$ and a priori estimation of the mean local vessel and background intensities, $I_{v_{i-1}}$ and $I_{b_{i-1}}$ respectively, geometrical moments allow estimating the local center $P_{i}^{\prime}$ and radius $r_{i}$ (see Fig. 3). For the first position $P_{0}$, the user has to specify the initial size of the spherical window $R_{0}^{0}, I_{v_{0}}$ and $I_{b_{0}}$. Then, for any point $P_{i}$, the initial size of the spherical window $R_{i}^{0}$ is set with respect to the previous local radius $r_{i-1} . I_{v}$ and $I_{b}$ are estimated using an expectation maximization (EM) algorithm. A histogram computed on an extended region (including the vessel and the background) around the point $P_{i-1}$ is modelized by two Gaussians. The background intensity $I_{b}$ (respectively the vessel intensity $I_{v}$ ) is considered as the mean of the Gaussian corresponding to the lowest intensities (respectively the highest).

\section{Parameter tuning}

Several parameters of the algorithm must be tuned: the vesselness filter parameters $(\alpha, \beta$ and $\gamma)$ and the thresholds controlling the orientation constraint of the front $(\mu, r$ and the Freezing distance $d$ ). Experiments were performed on synthetic data to study the influence of these parameters. The following tests were executed on a $1.6 \mathrm{GHz}$ Xeon PC, $2 \mathrm{~GB}$ RAM. The algorithms were implemented in $\mathrm{C}++$.

\section{A. Numerical Phantom Description}

An isotropic synthetic data set of size $110 \times 90 \times 90$ voxels has been designed from the VTK library which represents a part of the coronary venous tree with typical nearby structures such as cardiac cavities and coronary arteries. The contrast features of coronary veins in data sets are simulated to have the same density distribution as in real data. From here on

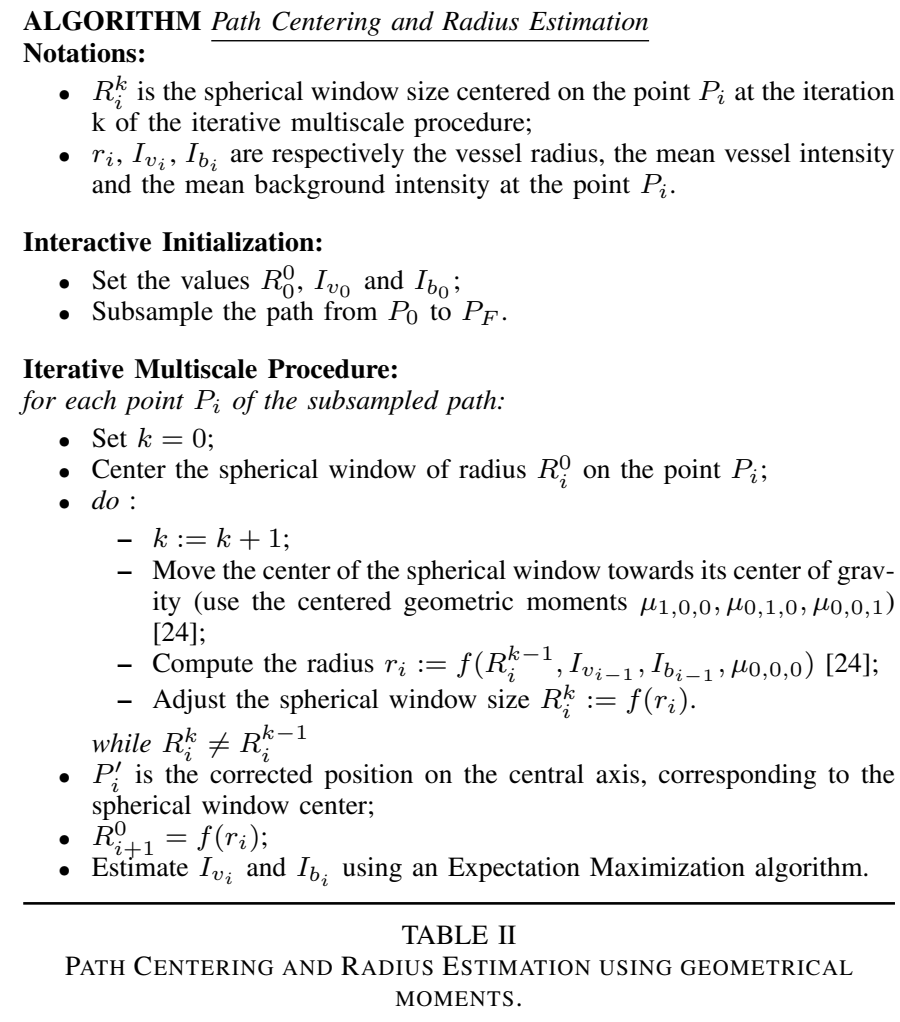

we will refer to this data set as 'phantom'. Fig. 4 depicts a surface representation of the phantom. For geometry simplicity, the coronary venous tree model is based on coronary vein centerlines extracted from real data (Coronary Sinus (CS), Great Cardiac Vein (GCV), Antero Lateral Vein (ALV)). This model that includes a bifurcation, consists of a tortuous tubular structure of length similar to real veins $(72,8 \mathrm{~mm})$. The main vessel radius varies from 6 to 1 voxels $(1$ voxel $\approx 0.4 \mathrm{~mm}$ ), and the second branch from the bifurcation has a constant radius of 1 voxel. Furthermore, a spherical structure represents a nearby cardiac cavity and cylinders of constant radius (4 voxels) represent crossing coronary artery segments (typical coronary radius varies from 1 to $4,5 \mathrm{~mm}$ ).

A curvilinear reconstruction along the main vessel axis is shown in Fig. 5(a) to visualize the intensity grey value distribution. As regards the densities laying inside the tube, difficulties due to the dye product have been considered. A succession of hypo-, hyper- and iso-dense areas was set in the range $[100-300]$ Hounsfield Units (HU). The sphere and cylinders density was set to $250 \mathrm{HU}$. As regards background density, since coronary veins are directly in contact with the adipose epicardic tissue, the value was set to $-80 \mathrm{HU}$ (mean density of adipose epicardic tissue on Hounsfield scale). The whole data set was finally smoothed using a Gaussian kernel to make contours blurred, and thus to get a local intensity profile similar to those on real data sets. Furthermore, to cope with real data noise, we added Gaussian noise to the data set. Thus, six data sets with different standard deviation $\sigma_{\text {noise }}=[0,10,20,30,40,50]$ are available for testing. Fig. 5 depicts the intensity distribution for the six configurations. 


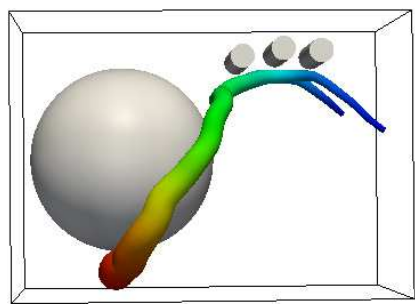

(a)

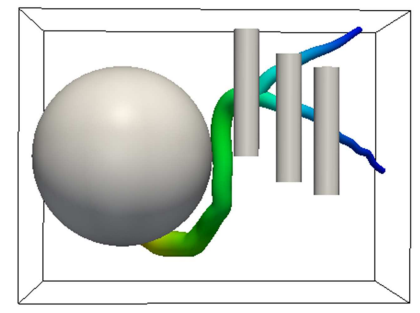

(c)

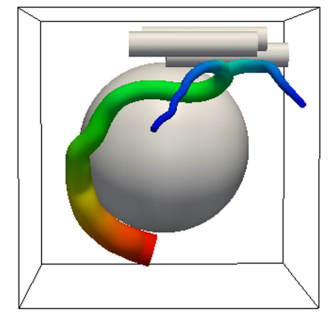

(b)

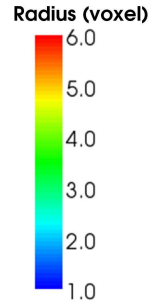

(d)
Fig. 4. Surface rendering of the synthetic data set under three different views (a)-(c): A tortuous tubular structure with a bifurcation representing a part of the coronary venous tree and exhibiting a radius varying from 1 to 6 voxels, a spherical structure representing a cardiac cavity and cylinders of constant radius (4 voxels) representing crossing coronary artery segments. (a) $\sigma_{\text {noise }}=0$

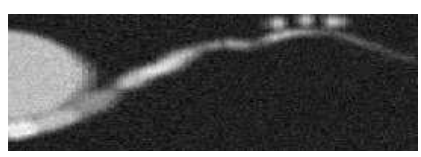

(c) $\sigma_{\text {noise }}=20$

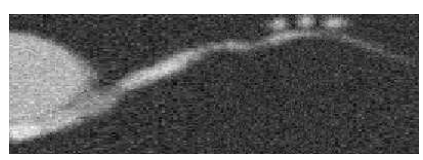

(e) $\sigma_{\text {noise }}=40$

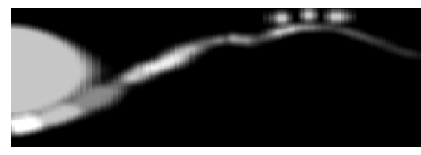

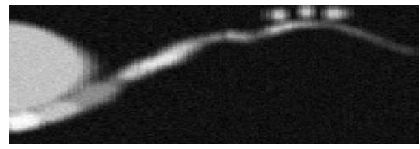

(b) $\sigma_{\text {noise }}=10$

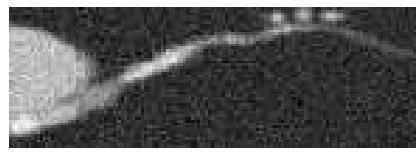

(d) $\sigma_{\text {noise }}=30$

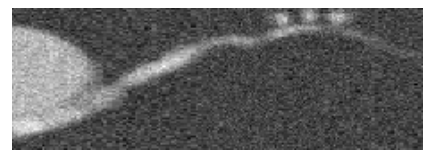

(f) $\sigma_{\text {noise }}=50$
Fig. 5. Curvilinear reconstruction along the main vessel axis of the model for the six configurations of the numerical phantom with $\sigma_{\text {noise }}$ varying from 0 to 50: Succession of hypo-, hyper- and iso-dense areas in the range [100 - 300] Hounsfield Units (HU).

\section{B. Vesselness Filter Parameters}

Frangi's filter parameters are $\alpha, \beta$ and $\gamma$. Olabarriaga et al. [25] evaluated the previous parameters effect on coronary arteries axis enhancement from CT. They demonstrated the quasi insensitivity of the parameters $\alpha$ and $\beta$ on filter response, whereas the background noise suppression term $\gamma$ has a strong impact on it. Furthermore, $\gamma$ relies on the grey-value distribution present in the image [21]. We set $\alpha$ and $\beta$ both to 0.5 as in Frangi's work [21] and performed series of test on the parameter $\gamma$.

We applied the vesselness filter for a fixed scale $\sigma=4$, with varying values of $\gamma$, on each of the six configurations of the phantom $\left(\sigma_{\text {noise }}=[0,10,20,30,40,50]\right)$. In order

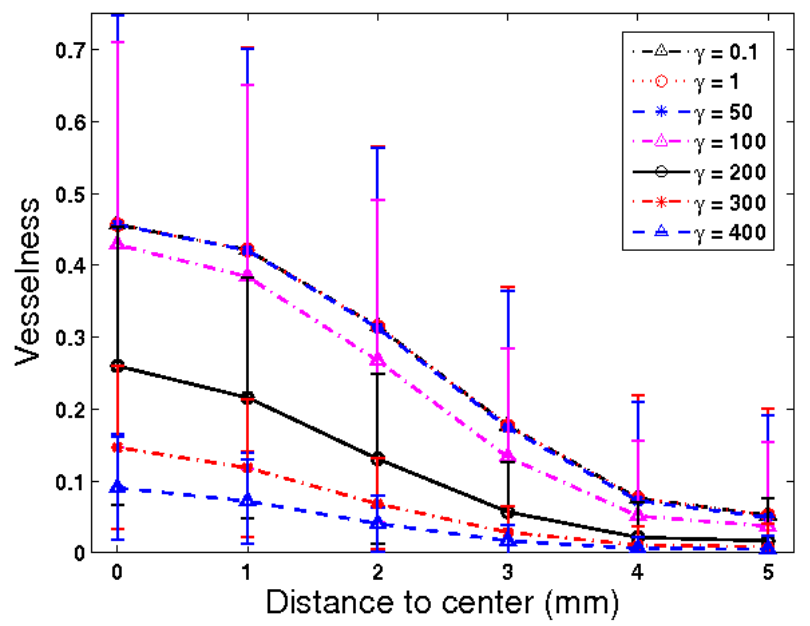

Fig. 6. Vesselness measure at different distances to center of the vessel for $\sigma_{\text {noise }}=50$.

to only study the influence of the parameter $\gamma$, the tubular structure radius was set to a constant value similar to $\sigma$ (4 voxels). Fig. 6 displays the mean vesselness measure (along the vessel) at different distances to center [0 - 5 voxels] for $\gamma=[0.1,1,50,100,200,300,400]$ and for $\sigma_{\text {noise }}=50$. Similar curves were obtained for the other values of $\sigma_{\text {noise }}$, e.g., for $\gamma=50$ at position 0 , the mean vesselness measure varies from 0.451 to 0.456 . Indeed, the different noisy data are based on the same noise-free data and the contrast with the background along the vessel trajectory is similar. Thus, we can say that the Frangi's filter is very successful in removing the background noise.

We are looking for values of $\gamma$ which yield high response close to axis (position 0) and an important slope between axis and contours (position 4). This is the case for $\gamma \in[0.1-50]$ (plots superimposed in the figure). We finally chose $\alpha=0.5$, $\beta=0.5$ and $\gamma=50$ for the following part.

\section{Orientation Constraint Parameters}

The second experiment consists in studying the influence of the parameters controlling the orientation constraint of the Fast-Marching front propagation i.e., $\mu, r$ and the Freezing distance $d$. We applied our minimum cost path algorithm with the Fast-Marching front propagation (see II-A) to evaluate the impact of each parameter on our six configurations of the phantom (noise and noise free configurations). We made the parameter $\mu$ vary within the interval $[0.5-0.9]$ with a step of $0.1, r$ within $[65-90]$ degrees with a step of 5 and the Freezing distance $d$ within $[6-12]$ with a step of 2 . For each combination of these parameters, an OVerlap score OV was computed to evaluate the extraction ability of the algorithm. This metric measures a point-to-point correspondence between the phantom centerline (ground truth) and the extracted centerline and provides a score between 0 and 1 (more details about the OV metric computation can be found in [19]). More the extracted centerline follows the phantom centerline, closer to 1 the score is. High OV scores $(0.76-0.90)$ were obtained for combinations of $\mu, r$ and $d$ respectively lying in the range 
$[0.7-0.75],[80-90]$ and $[6-12]$. The Freezing distance $d$ was not found to be a sensitive parameter. Indeed, the cost function guides the front propagation whereas the Freezing procedure aims at blocking the propagation at the front tail beyond a given distance. So based on these results, we chose $\mu=0.75$ et $r=80$ degrees and for the freezing distance, we applied a value close to the vessel radius.

\section{Evaluation and Results on Venous Data Sets}

\section{A. MSCT DATA Description}

Experiments have been carried out on three MSCT sequences of patients candidates to CRT. Imaging was performed with a General Electric LightSpeed VCT 64-slice Scanner. The same protocol was used for all the acquisitions, that is a contrast agent injection in the coronary tree and a prolonged scan delay compared to coronary imaging in cardiac MSCT. Each sequence included 20 volumes reconstructed from slices acquired every $5 \%$ of the R-R interval from the cardiac cycle. There are approximately 200 slices per volume, with $512 \times 512$ pixels per slice. The resolution is about $0.4 \times 0.4 \mathrm{~mm}^{2}$ per pixel. Since the interslice space is higher than the pixel size, a preliminary interpolation was performed to make the data sets isotropic.

\section{B. Coronary Vein Visibility}

As explained in the introduction, scanning protocols are tailored for optimal visualization of the coronary arteries. As a consequence, the coronary venous system exhibits a weak contrast is barely contrasted, vascular branches are located within a noisy multi-object environment and their appearance varies along the tree and over time. In addition, artifacts are present in some volumes due to reconstruction problems. They can be either caused by an irregular heart rate or by too fast motions of structures related to the rotation speed of the acquisition system. These artifacts mainly occur in early systolic and end-diastolic phases, and can affect $50 \%$ of the volumes of the sequence. Motion artifacts induce blurring effects and a splitting or duplication of vessels, making their extraction difficult, even impossible, in some cases.

Thus, we asked a medical expert (who is an interventional cardiologist) to qualify the venous branches for each volume of the considered sequences, according to their quality in terms of contrast or visibility and provide for each, their name and their location. He was able to only classify vessels in volumes ranging from $45 \%$ to $85 \%$ of the R-R interval from the cardiac cycle which approximately corresponds to the diastolic phase (its duration varies from patient to patient). He labelled thus the coronary venous branches on eighteen volumes from the three sequences and classified 114 veins according to their visibility (Table III). Four categories were considered: High visibility (++), good visibility $(+)$, medium visibility (+-) and low visibility (--). High visible vessels exhibit homogeneous high density with strong edges such as in Fig. 7(a) and 7(b). In case of good visibility (Fig. 7(c) and 7(d)) and medium visibility (Fig. 7(e) and 7(f)), the contrast filling is inhomogeneous with hypodense zones, attachment of nearby structures and blurred contours. Finally, in Fig. 7(c) and (a) CS (++)

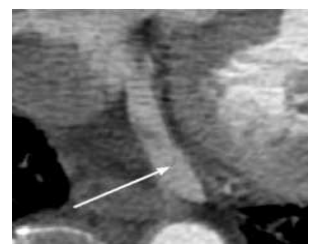

(c) $\mathrm{CS}(+)$

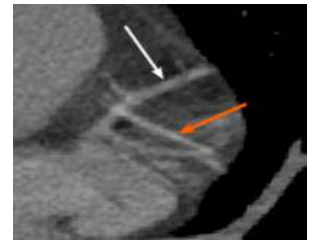

(e) GCV (+-)

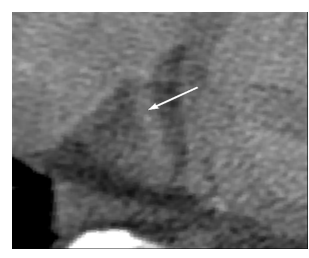

(g) CS (--)

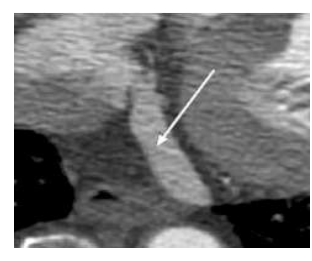

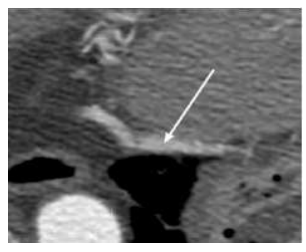

(b) PLV (++)

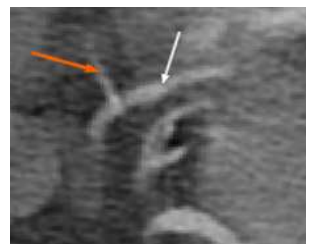

(d) $\operatorname{MCV}(+)$

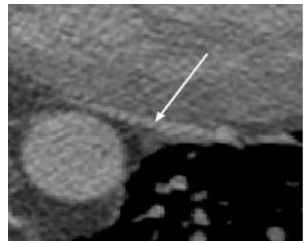

(f) $\mathrm{PV}(+-)$

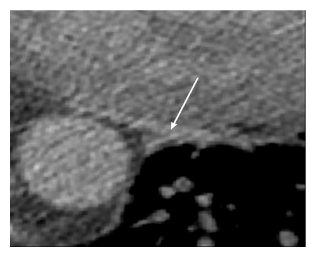

(h) PV (--)
Fig. 7. Exemples of coronary vein (white arrow) permeabilities on CT slices: High visibility $(++)$, good visibility $(+)$, medium visibility $(+-)$ and low visibility (--). Crossing coronary arteries are indicated with an orange arrow.

\begin{tabular}{|c|c|c|c|c|}
\hline Vessel Visibility & ++ & + & +- & -- \\
\hline Coronary Sinus (CS) & 11 & 11 & 9 & 5 \\
\hline Middle Cardiac Vein (MCV) & $/$ & 6 & 6 & 6 \\
\hline Posterior or Postero-Lateral Vein (PV/PLV) & 2 & 4 & 9 & 8 \\
\hline Great Cardiac Vein (GCV) & $/$ & 2 & 11 & 5 \\
\hline Antero or Antero-Lateral Vein (AV/ALV) & $/$ & 2 & 7 & 10 \\
\hline
\end{tabular}

TABLE III

PHYSICIAN EXPERTISE: CLASSIFICATION OF 114 CORONARY VEINS ACCORDING TO THEIR NAME AND VISIBILITY INTO THE EIGHTEEN MSCT VOLUMES. THESE VOLUMES ARE THOSE WHICH ARE NOT POLLUTED WITH MOTION ARTIFACTS OVER THE SET OF THE THREE SEQUENCES. EACH COLUMN DISPLAYS THE NUMBER OF SEGMENTS IN EACH CATEGORY (FROM HIGH TO LOW VISIBILITY

7(d) we can observe low visible vessels with similar density to that of adjacent tissues.

The Coronary Sinus (CS) is generally highly visible. The Middle Cardiac Vein (MCV) may be well visible in some volumes as well as poorly contrasted in other volumes. The Posterior (PV) and Postero-Lateral (PLV) Veins more often exhibit medium or low visibility, with a few cases of good visibility. The Great Vein (GV) is mainly visible in most cases. Finally, the Antero (AV) and Antero-Lateral (ALV) Veins tend to be poorly visible. Those degrees of visibility are also correlated with vessel caliber (measures of the CS and its 
tributaries can be found in [5]). Indeed, the CS exhibits a big diameter along its trajectory, and becomes smaller to form the Great Vein. Thinner calibers are observed for the Antero and Antero-Lateral Veins.

\section{Extraction Results}

In order to stimulate the left ventricle, the target vein is ideally located on the lateral wall. If any ALV cannot be reached by the cardiologist, the second choice of implantation is a PV or the MCV and the GV at last. In the context of interventional planning, it is of interest to visualize in 3D space the possible implantation paths (see Fig. 8), but also to observe accurately the extracted branches with their caliber information and the nearby structures on curvilinear reconstructions (see Fig. 9).

We applied thus the algorithm on the real data sets, using the parameters designed in section III: $\alpha=0.5, \beta=0.5$, $\gamma=50$ for Frangi's filter and $\mu=0.75, r=80$ degrees and the freezing distance $d=12$ for the Fast-Marching front propagation. Frangi's filter was applied at different scales in order to cope with vessels of different calibers. Since the coronary venous tree exhibits highly different diameters, we made the scales vary from 1 to 4 . Limiting the highest scale to 4 , prevented the filter from yielding false positive response beyond contours, which is essential in low-contrast environment.

Extraction results obtained on two patient data sets are shown in Fig. 8 (identified as cases Patient 1 and Patient 2). From user-defined points, the algorithm extracts first the coronary sinus and the great cardiac vein in its continuity. Then, each contributing branch is extracted in turn, which yields immediately a tree shape with bifurcation positions. For Patient 1 , the whole coronary venous tree has been extracted from four initialization points, into two consecutive volumes (50\% and 55\%): the optimal path from CS to ALV and an alternative path through the MCV (see Fig. 8(a)). During the CRT procedure, the LV lead has been implanted in the distal part of the MCV. For Patient 2, the whole coronary venous tree has been extracted from four initialization points, into three consecutive volumes $(50 \%, 55 \%$ and $60 \%)$ : the optimal path from CS to ALV and an alternative path through the MCV (see Fig. 8(b)). During the CRT procedure, the LV lead has been implanted in the distal part of the ALV.

We can observe on the curvilinear reconstructions (Fig. 9), the method ability to accurately extract tubular structures of different calibers (1.0 to $9.3 \mathrm{~mm}$ ) and structures presenting contrast difficulties. In Fig. 9(a) and 9(c), we can observe vessel segment whose densities overlap with those of nearby structures (circled in red on the image). In Fig. 9(e), the CS exhibits an abrupt change in diameter due to an area very thin and poorly contrasted (circled in red on the image).

\section{Qualitative Evaluation}

We compared our minimum cost path algorithm (VM + OC) with a tracking technique developed by [24]. Moreover for our algorithm, we considered as a comparison, three different cost functions to build the travel time map: (1) the intensity

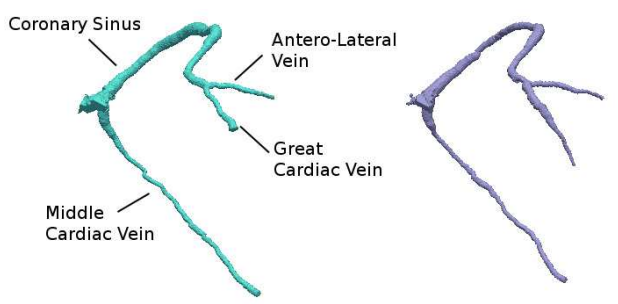

(a) Patient 1: Volumes 50\%-55\%

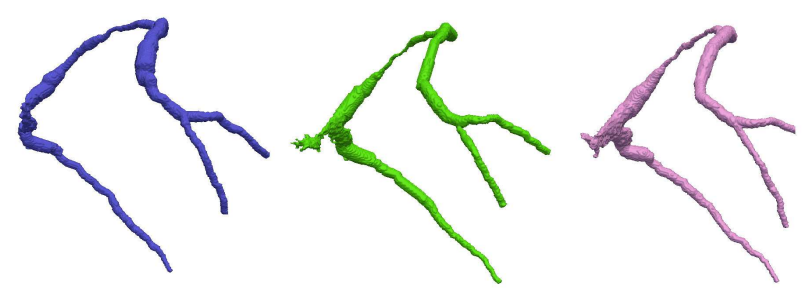

(b) Patient 2: Volumes 50\%-55\%-60\%

Fig. 8. 3D Extraction of the coronary veins of interest for LV lead implantation.

information that makes the assumption that vessels are brighter than surrounded structures (method I - was applied in [15] to extract the trachea, brain vessels and an aorta), (2) the hessian-based vesselness measure (method VM - was applied in [26] to extract coronary arteries) in order to discriminate tubular structures from blob- or plate-like structures, (3) the combination of the multiscale Frangi's filter response with the direction of the front with respect to the vessel orientation (our method VM + OC).

For method I, the cost function was defined as follows [15]:

$$
\Lambda(x, y, z)=\left|I_{\text {mean }}-I_{x, y, z}\right|^{2}
$$

where $I_{\text {mean }}$ is the mean lumen intensity of the vessels of interest and $I_{x, y, z}$ the intensity at position $(x, y, z)$.

The second one (method VM) was given by [26]:

$$
\Lambda(x, y, z)= \begin{cases}\frac{1}{F_{x, y, z}} & \text { if } F_{x, y, z}>s \\ C, & \text { otherwise }\end{cases}
$$

where $F_{x, y, z}$ is the Frangi's vesselness measure at position $(x, y, z)$ and $C$ is the cost given at non-vessel positions (threshold $s$ ). $C$ and $s$ have similar values to those used in method $(\mathrm{VM}+\mathrm{OC})$. The three methods $(\mathrm{VM}+\mathrm{OC}),(\mathrm{VM})$ and (I) are applied with the identical Freezing procedure (distance $d$ set to 12 voxels) and the same Frangi's filter parameters $(\alpha=0.5, \beta=0.5$ and $\gamma=50)$.

The tracking algorithm (referred as method Mom) relies on a local modelling of the vessel by a cylinder in a 3D homogeneous space. The main advantage of this method is to provide analytical expressions based on 3D geometrical moments for the computation of the local cylinder parameters (location of the center of gravity, local orientation and diameter). The initialization step is performed interactively by pointing the vascular branch of interest. The tracking is then performed by shifting a spherical window according to the estimated orientation and the exploration is carried out in the two opposite directions. Nevertheless, the orientation estimated at a given position does not guarantee an accurate 

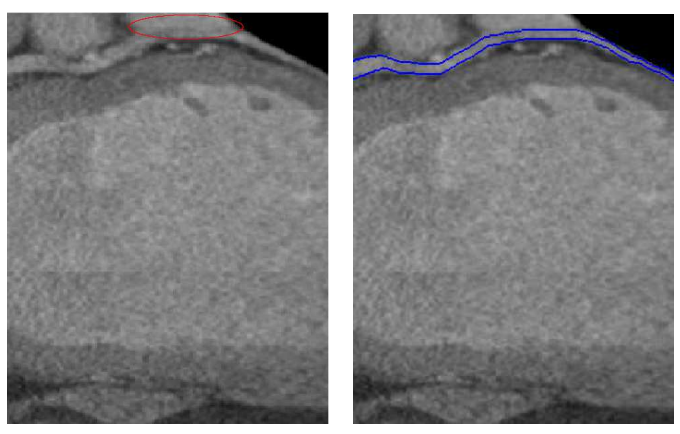

(a) GCV (Patient 1 vol. 55\%) (b) GCV + radius estimation
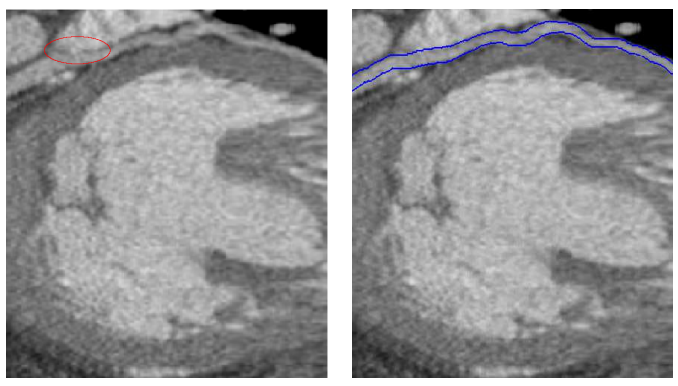

(c) ALV (Patient 2 vol. 50\%)

(d) ALV + radius estimation

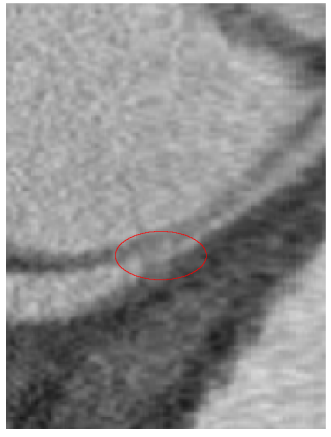

(e) CS (Patient 2 vol. 50\%)

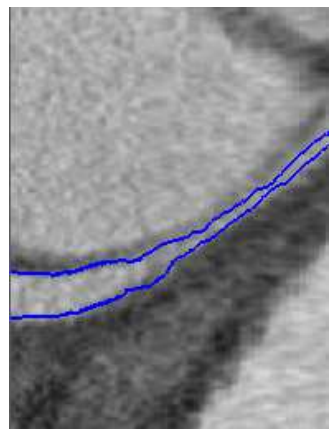

(f) $\mathrm{CS}+$ radius estimation
Fig. 9. Curvilinear representations from extracted centre lines (low contrast areas circled in red).

\begin{tabular}{|c|c|c|c|c|}
\hline Vessel Visibility & ++ & + & +- & -- \\
\hline Method (VM + OC) & $100 \%$ & $88 \%$ & $83.3 \%$ & $47.1 \%$ \\
\hline Method (VM) & $100 \%$ & $88 \%$ & $71.4 \%$ & $32.3 \%$ \\
\hline Method Mom & $84.6 \%$ & $44 \%$ & $35.7 \%$ & $17.6 \%$ \\
\hline
\end{tabular}

TABLE IV

PERCENTAGE OF SUCCESSFUL PATH EXTRACTIONS FOR EACH METHOD ACCORDING TO CORONARY VEIN VISIBILITY IN MSCT DATA SETS.

position of the next point on the central axis in particular when highly curved vessels are tracked. An iterative centering process (see Table II) is therefore achieved, using the first order moments, to move the point towards the vessel center. The incremental displacement between two tracked points is adaptive and depends on the vessel size and curvature. In most cases, and especially for coronaries, the tissue surrounding the vessels is not homogeneous. The presence of different tissues in the immediate neighbouring of the vessels is detected and an EM algorithm is applied to estimate for each point the local mean vessel and background intensities.

We studied the ability of each method to extract the vein central axis from an initial point to a final point (without interactive re-initialization). Each method has been applied to the 114 veins classified according to their visibility in MSCT volumes (see Table III).

The percentage of successful extracted path is given in Table IV. An extraction error means that the algorithm did not reach the final point of the branch. The method (I) based on intensity information fails in the proximal part of each branch (result are not reported in Table IV). The contrast inhomogeneities in the vessel lumen lead the front towards wrong directions. The method Mom exhibits a quite good score for images of good visibility. This score quickly decreases with the image corruption. This is due to the fact that the multiscale recentering process makes the center of the spherical window move towards brightest structures. As the contrast inside the veins is often weaker than its close environment (cardiac cavities, myocardium, arteries), the tracking algorithm tends to jump into these neighbouring structures. The methods based on a vesselness measure (VM and $\mathrm{VM}+\mathrm{OC}$ ) appear globally more successful. Indeed, we can notice the very high and similar scores for veins of high and good visibility for the methods $(\mathrm{VM}+\mathrm{OC})$ and $(\mathrm{VM})$. Finally, the introduction of the orientation constraint in the cost function $(\mathrm{VM}+\mathrm{OC})$ allow to obtain the best results for veins of medium and low visibility.

\section{CONClusion}

An algorithm was proposed to deal with coronary vein extraction in MSCT volumes. Difficulties are related to the complexity of the scene that includes different structures such as the myocardium, the cavities and the coronary venous and arterial trees, which are very close to each other and have a similar contrast. Moreover, due to the MSCT scan protocol, the venous tree is not well contrasted and its appearance can be highly variable. One more difficulty comes from the cardiac beat that introduces in some volumes (especially those associated with the systolic and early-diastole phases) motion artifacts that blur the structures, making the extraction of the veins in these volumes impossible. As regards the minimum cost path method, we proposed a specific cost function, which allows to constrain a front propagation in the local vessel orientation. Parameter setting was performed on simulated data to find the optimal values based on different criteria. The qualitative analysis based on the vascular visibility and the comparison to three other methods shows a good efficiency of the algorithm in a low-contrast environment with a fairly good accuracy in the venous branch extraction. In the context of CRT, the method allows to describe the patient-specific coronary venous tree with the optimal implantation path as well as alternative ones. The proposed approach could be used pre-operatively to actually plan the catheterization procedure by mapping the possible $3 \mathrm{D}$ access paths. It could contribute to optimize the CRT procedure. Further work is needed to confirm the method robustness on more patient cases. In future projects, those results could be used as input data for catheterism simulation system and registered with intraoperative data for interventional assistance. 


\section{REFERENCES}

[1] C. Leclercq and D. Kass, "Re-timing the failing heart: Principles and current clinical status of cardiac resynchronization," J. Am. Coll. Cardiol., vol. 39, no. 2, pp. 194-201, 2002.

[2] J. Daubert, P. Ritter, H. L. Breton, D. Gras, C. Leclercq, A. Lazarus, J. Mugica, P. Mabo, and S. Cazeau, "Permanent left ventricular pacing with transvenous leads inserted into the coronary veins," PACE, vol. 21, pp. 239-245, 1998 .

[3] W. T. Abraham, W. G. Fisher, A. L. Smith, D. B. Delurgio, A. R Leon, E. Loh, D. Z. Kocovic, M. Packer, A. L. Clavell, D. L. Hayes, M. Ellestad, R. J. Trupp, J. Underwood, F. Pickering, C. Truex, P. McAtee, and J. Messenger, "Cardiac resynchronization in chronic heart failure." $N$ Engl J Med, vol. 346, no. 24, pp. 1845-1853, Jun 2002.

[4] R. Mlynarski, M. Sosnowski, A. Wlodyka, W. Kargul, and M. Tendera, "A user-friendly method of cardiac venous system visualization in 64-slice computed tomography." Pacing Clin Electrophysiol, vol. 32, no. 3, pp. 323-329, Mar 2009. [Online]. Available: http://dx.doi.org/10.1111/j.1540-8159.2008.02239.x

[5] J. R. Ortale, E. A. Gabriel, C. Iost, and C. Q. Marquez, "The anatomy of the coronary sinus and its tributaries." Surg Radiol Anat, vol. 23, no. 1, pp. 15-21, 2001.

[6] J. Suri, K. Liu, L. Reden, and S. Laxminarayan, "A review on MR vascular image processing: skeleton versus nonskeleton appoaches: part II," IEEE Trans. Inf. Technol. Biomed., vol. 6, no. 2, pp. 142-158, 2002.

[7] C. Kirbas and F. K. H. Quek, "A review of vessel extraction techniques and algorithms," ACM Comput. Surv., vol. 36, no. 2, pp. 81-121, 2004

[8] D. Lesage, E. D. Angelini, I. Bloch, and G. Funka-Lea, "A review of 3D vessel lumen segmentation techniques: models, features and extraction schemes." Med Image Anal, vol. 13, no. 6, pp. 819-845, 2009.

[9] J. Yi, "A locally adaptive region growing algorithm for vascular segmentation," Int. J. Imaging Syst. Technol., vol. 13, no. 4, pp. 208-214, 2003.

[10] T. McInerney and D. Terzopoulos, "T-snakes: topology adaptive snakes." Med Image Anal, vol. 4, no. 2, pp. 73-91, Jun 2000.

[11] J. Sethian, Level Set Methods and Fast Marching Methods: Evolving Interfaces in Computational Geometry, Fluid Mechanics, Computer Vision and Materials Science, 2nd ed. Cambridge University Press, 1999.

[12] C. Toumoulin, C. Boldak, J. L. Dillenseger, J. L. Coatrieux, and Y. Rolland, "Fast detection and characterization of vessels in very large 3-D data sets using geometrical moments," IEEE Transactions on Biomedical Engineering, vol. 48, no. 5, pp. 604-606, 2001.

[13] J. Velut, C. Toumoulin, and J.-L. Coatrieux, "3D coronary structure tracking algorithm with regularization and multiple hypotheses in MRI," in Proc. IEEE Int Biomedical Imaging: From Nano to Macro Symp, 2010, pp. 37-40.

[14] W. Wong and A. Chung, "Augmented vessels for quantitative analysis of vascular abnormalities and endovascular treatment planning," IEEE Trans. Med. Imaging, vol. 25, no. 6, pp. 665-684, 2006.

[15] T. Deschamps and L. Cohen, "Fast extraction of minimal paths in 3D images and applications to virtual endoscopy," Medical Image Analysis, vol. 5, no. 4, pp. 281-299, 2001.

[16] Q. Lin, "Enhancement, Extraction, and Visualization of 3D Volume Data," Institute of Technology, department of Electrical Engineering, Linkoping University, Sweden, 2003.

[17] L. Cohen and T. Deschamps, "Segmentation of 3D tubular objects with adaptive front propagation and minimal tree extraction for 3D medical imaging," Computer Methods in Biomechanics and Biomedical Engineering, vol. 10, no. 4, pp. 289-305, 2007.

[18] H. E. „Cetingül, M. A. Gülsün, and H. Tek, "A unified minimal path tracking and topology characterization approach for vascular analysis," Medical Imaging and Augmented Reality, Lecture Notes in Computer Science, vol. 6326, pp. 11-20, 2010.

[19] C. Metz, M. Schaap, T. van Walsum, A. van der Giessen, A. Weustink, N. Mollet, G. Krestin, and W. Niessen, "3D Segmentation in the Clinic: a Grand Challenge II - Coronary Artery Tracking," in MICCAI, 2008.

[20] M.-P. Garcia, C. Toumoulin, P. Haigron, J. Velut, M. Garreau, and D. Boulmier, "Coronary vein tracking from MSCT using a minimum cost path approach." in Proceedings of the IEEE International Symposium on Biomedical Imaging: From Nano to Macro, 2010, pp. 17-20.

[21] A. Frangi, W. Niessen, K. Vincken, and M. Viergever, "Multiscale vessel enhancement filtering," MICCAI, pp. 130-137, 1998.

[22] T. Deschamps and L. D. Cohen, "Fast Extraction of Tubular and Tree 3D Surfaces with front propagation methods," in 16th International Conference on Pattern Recognition, ICPR'02, 2002.
[23] G. Parker, C. Wheeler-Kingshott, and G. Barker, "Estimating distributed anatomical connectivity using fast marching methods and diffusion tensor imaging," IEEE Transactions on Medical Imaging, vol. 21, no. 5, pp. 505-512, 2002.

[24] C. Boldak, Y. Rolland, C. Toumoulin, and J.-L. Coatrieux, "An improved model-based vessel tracking algorithm with application to computed tomography angiography." Biocybern. Biomed. Eng., vol. 23, pp. 4163, 2003.

[25] S. Olabarriaga, M. Breeuwer, and W. Niessen, "Evaluation of Hessianbased filters to enhance the axis of coronary arteries in CT images," Computer Assisted Radiology and Surgery, vol. 1256, pp. 1191-1196, 2003.

[26] - "Minimum Cost Path Algorithm for Coronary Artery Central Axis Tracking in CT Images," MICCAI (2), pp. 687-694, 2003. 